\title{
The association between pulse pressure and vascular access thrombosis in chronic hemodialysis patients
}

\author{
Che-Yi Chou, Jiung-Hsiun Liu, Huey-Liang Kuo, Yao-Lung Liu, Hsin-Hung Lin, Ya-Fei Yang, Shu-Ming Wang \\ and Chiu-Ching Huang
}

Vascular access thrombosis (VAT) is a major cause of morbidity in chronic hemodialysis (HD) patients and is characterized by chronic inflammation. Pulse pressure (PP) is positively associated with chronic inflammation. Whether patients with high PP are at a higher risk for VAT, however, remains unknown. We retrospectively reviewed chronic HD patients with a functional vascular access point at the China Medical University Hospital between 1986 and 2005. The association between PP and the initial development of VAT at the primary vascular access point was examined using the Kaplan-Meier analysis and multivariate Cox proportional hazards regression. A total of 576 chronic HD patients (264 men and 312 women) with a mean age of $56.9 \pm 14.0$ years were reviewed, of whom 145 (25.2\%) experienced at least one episode of VAT. Patients with a PP $>60 \mathrm{~mm} \mathrm{Hg}$ had a lower VAT-free survival rate compared with those with a PP $<60 \mathrm{~mm} \mathrm{Hg}(P<0.001)$. Using Cox regression with adjustments for age, systolic blood pressure and vascular access types, PP (every increase of $10 \mathrm{~mm} \mathrm{Hg}$ ) and serum C-reactive protein (CRP) (every increase of $1 \mathrm{mg}$ per $100 \mathrm{ml}$ ) were found to be independently associated with an increasing risk for VAT, with a hazard ratio of 2.57 (95\% confidence interval: $1.5-4.4, P=0.001$ ) and 1.14 (95\% confidence interval: $1.01-1.27, P=0.017$ ), respectively. High PP was associated with the development of VAT in chronic HD patients. This association was independent of serum CRP levels.

Hypertension Research (2009) 32, 712-715; doi:10.1038/hr.2009.88; published online 10 July 2009

Keywords: atherosclerosis; diabetes; hemodialysis; pulse pressure; vascular access thrombosis

\section{INTRODUCTION}

Vascular access thrombosis (VAT) is one of the most common causes of morbidity and is the major cause of hospitalization in chronic hemodialysis (HD) patients. ${ }^{1}$ In the traditional view, neointimal hyperplasia and luminal stenosis are the keys to the development of VAT. ${ }^{1}$ Mounting evidence has shown that VAT is associated with an increase in systemic inflammation markers, such as C-reactive protein $(\mathrm{CRP})^{2}$ and homocysteine, ${ }^{3,4}$ in chronic HD patients. An increase in inflammatory markers, such as macrophages, lymphocytes, vascular cell adhesion molecule- 1 , tumor necrosis factor- $\alpha$ and interleukin- 6 , is found on primary stenotic lesions. ${ }^{5}$ This suggests the chronic inflammatory nature of VAT.

Elevated pulse pressure (PP) is associated with an increase in overall mortality and cardiovascular events in chronic HD patients. ${ }^{6,7}$ Several specific measurements of $\mathrm{PP}$, such as central $\mathrm{PP}^{8}$ and nocturnal $\mathrm{PP},{ }^{9}$ are also associated with increasing mortality and cardiovascular disease in patients with end-stage renal disease. PP is positively associated with an increase in $\mathrm{CRP}^{10}$ and other inflammatory markers, such as intercellular adhesion molecule- 1 and interleukin-6. ${ }^{11}$ Every $10-\mathrm{mm} \mathrm{Hg}$ increment of $\mathrm{PP}$ is associated with a $22 \%$ increase in cardiovascular risk and systolic blood pressure (SBP), resulting in a
$10 \%$ increase in cardiovascular risk. ${ }^{12} \mathrm{PP}$, serum CRP and plasma homocysteine are positively associated with an increase in atherosclerotic plaques and intima-media thickness in uremic patients. ${ }^{13}$ Given that VAT and elevated PP are associated with a chronic inflammatory condition, it is possible that elevated PP is associated with the development of VAT in chronic HD patients. We conducted this study to determine the association between PP and the development of VAT in chronic HD patients.

\section{METHODS}

All chronic HD patients dialyzed for more than 3 months (in three 4-h sessions per week) between 1986 and 2005 at the China Medical University Hospital were reviewed. A total of 576 patients dialyzed through a primary functional vascular access point, including native arteriovenous fistula and arteriovenous graft (AVG), were included; 363 patients with temporary vascular access and a history of non-functional vascular access were excluded. SBP and diastolic blood pressure (DBP) were measured monthly, with the patient in the supine position before $\mathrm{HD}$ sessions, and the 3-month average blood pressure was used for analysis. PP was calculated as SBP-DBP. The mean arterial pressure was calculated as $(\mathrm{SBP}+2 \times \mathrm{DBP}) / 3$. The duration of vascular access was recorded from the initiation of $\mathrm{HD}$ treatment through the functional vascular access point to the date of the first VAT episode or December 2005. This duration was 
shortened by death, on being shifted to peritoneal dialysis, on undergoing kidney transplantation or being transferred to another HD center. A VAT event was defined as a sudden cessation of function of the vascular access, rendering HD impossible and requiring a thrombectomy, thrombolysis or acute placement of another HD access. ${ }^{2}$

Biomarkers including hematocrit, creatinine, uric acid, albumin, cholesterol, triglyceride, total calcium and phosphate were recorded every month. Intact parathyroid hormone and $K_{\mathrm{t}} / V$ urea were recorded every 3 months. CRP was recorded at the initiation of $\mathrm{HD}$ and every year. For patients with more than two values of biomarkers available, the average value was used for analysis. Hypertension was defined as a history of blood pressure $>140 / 90 \mathrm{~mm} \mathrm{Hg}$ for $>2$ years that required the initiation of antihypertensive therapy by a primary physician. ${ }^{14}$ Diabetes mellitus was defined as a fasting glucose level of $140 \mathrm{mg}$ per $100 \mathrm{ml}$, a non-fasting glucose level of $200 \mathrm{mg}$ per $100 \mathrm{ml}$ or a history of or treatment for diabetes. ${ }^{15}$ Coronary arterial diseases-self-reported or evaluated in their medical records-included myocardial infarction, coronary artery bypass graft, percutaneous transluminal angioplasty, coronary stenosis greater than $50 \%$ and ischemic stroke at the beginning of $\mathrm{HD}^{16}$

\section{Statistical analysis}

All data were analyzed using a standard statistical package (SPSS for Windows, version 12; SPSS Inc., Chicago, IL, USA). Clinical and demographic data are reported as mean \pm s.d. or percentage frequency when appropriate. Student's $t$-test or the Mann-Whitney $U$-test was used for continuous variables and a $\chi^{2}$-test was used for categorical variables. A receiver-operating characteristic curve and the area under curve were created to identify multiple blood pressure measurements, including PP, SBP, DBP and mean arterial pressure, for the prediction of VAT. The VAT-free survival curve according to the patients' PP was analyzed using the Kaplan-Meier estimation. Factors that were significantly different between patients with and without VAT events were analyzed using univariate Cox regression. Factors with a $P$-value less than 0.05 and well-known risk factors such as age and coronary arterial disease were further analyzed using multivariate Cox regression. A $P$-value less than 0.05 was considered as statistically significant.

\section{RESULTS}

We retrospectively reviewed 576 chronic HD patients (264 men and 312 women) with a mean age of $56.9 \pm 14.0$ years. In an average follow-up time of 57.3 months, $145(25.2 \%)$ patients developed at least one episode of VAT. The demographic characteristics of the entire study population are shown in Table 1 . Of the patients who developed VAT, $46.9 \%$ had diabetes. This was significantly higher than the percentage of diabetic patients who did not have VAT $(33.9 \%$; $P=0.005)$. Patients who developed VAT had a higher SBP and PP than those who did not develop VAT $(P<0.001$ and $P<0.001$, respectively). Of our patients, $78.8 \%$ had a forearm native arteriovenous fistula (nAVF), 9.2\% had an upper-arm nAVF and $8.9 \%$ had a forearm AVG. Patients with VAT had a lower frequency of nAVF $\left(P<0.001, \chi^{2}\right.$-test $)$ and a higher frequency of upper-arm nAVF $(P<0.001)$ and forearm AVG $(P<0.001)$. In all, $17(18.6 \%)$ of 145 patients with VAT received warfarin, whereas only $3.2 \%$ of patients without VAT received warfarin $\left(P<0.001, \chi^{2}\right.$-test $)$. The biochemical characteristics of the entire study group are shown in Table 2. Patients who developed VAT had lower serum cholesterol levels and higher serum CRP levels compared with those who did not develop VAT $(P=0.003$ and $P=0.001$, respectively).

The receiver-operating characteristic curve and the area under curve for different blood pressure measurements and their ability to predict the development of VAT are shown in Figure 1. The areas under curve for PP and SBP were $0.613(P<0.001)$ and $0.588(P=0.001)$, respectively. The area under curve for DBP was $0.525(P=0.367)$ and that for mean arterial pressure was $0.555(P=0.048)$. The optimal cutoff for PP was $60 \mathrm{~mm} \mathrm{Hg}$, which is consistent with the cutoff suggested in
Table 1 Clinical and demographic characteristics of entire study population

\begin{tabular}{lccc}
\hline & VAT( $(-), \mathrm{n}=431$ & VAT $(+), \mathrm{n}=145$ & P-value \\
\hline Age (years) & $56.4 \pm 14.2$ & $58.3 \pm 13.4$ & 0.155 \\
Duration (months) & $62.1 \pm 36.7$ & $42.9 \pm 30.7$ & $<0.001$ \\
Male, $n(\%)$ & $188(43.6)$ & $76(52.4)$ & 0.06 \\
SBP $(\mathrm{mm} \mathrm{Hg})$ & $140.2 \pm 16.2$ & $145.7 \pm 15.8$ & $<0.001$ \\
DBP $(\mathrm{mm} \mathrm{Hg})$ & $80 \pm 7.9$ & $80.5 \pm 8.2$ & 0.508 \\
MAP $(\mathrm{mm} \mathrm{Hg})$ & $100.1 \pm 9.9$ & $102.2 \pm 9.9$ & 0.024 \\
PP $(\mathrm{mm} \mathrm{Hg})$ & $60.2 \pm 12.2$ & $65.2 \pm 11.6$ & $<0.001$
\end{tabular}

$\begin{array}{lrcl}\text { Underlying disease, } n \text { (\%) } & & & \\ \text { Diabetes } & 124(28.8) & 63(43.4) & 0.001 \\ \text { Hypertension } & 89(20.6) & 22(15.2) & 0.148 \\ \text { CGN } & 170(39.4) & 48(33.1) & 0.173 \\ \text { CIN } & 29(6.7) & 5(3.4) & 0.147\end{array}$

\section{Comorbidity}

$\begin{array}{lrrr}\text { Hypertension } & 201(46.6) & 77(53.1) & 0.178 \\ \text { Diabetes } & 146(33.9) & 68(46.9) & 0.005 \\ \text { CAD } & 71(16.5) & 31(21.4) & 0.181\end{array}$

$\begin{array}{lccc}\text { Native AVF } & & & \\ \text { Forearm } & 369(85.6) & 85(58.6) & <0.001 \\ \text { Upper } & 29(6.7) & 24(16.6) & <0.001\end{array}$

AVG

$\begin{array}{lccc}\text { Forearm } & 23(5.3) & 28(19.3) & <0.001 \\ \text { Upper } & 10(2.3) & 8(5.5) & 0.06\end{array}$

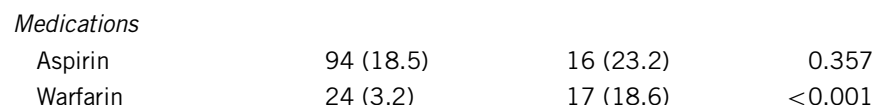

Abbreviations: AVF, arteriovenous fistula; AVG, arteriovenous graft; $C A D$, coronary artery disease; Abbreviations: AVF, arteriovenous fistula; AVG, arteriovenous graft; CAD, coronary artery diseas
CGN, chronic glomerular nephritis; CIN, chronic interstitial nephritis; DBP, diastolic blood pressure; PP, pulse pressure; SBP, systolic blood pressure; VAT, vascular access thrombosis.

Table 2 Biochemical characteristics of 576 chronic hemodialysis patients

\begin{tabular}{lccl}
\hline & VAT $(-), \mathrm{n}=431$ & VAT $(+), \mathrm{n}=145$ & P-value \\
\hline Hematocrit (\%) & $29.5 \pm 4.9$ & $29.6 \pm 4.5$ & 0.697 \\
Albumin (g per $100 \mathrm{ml})$ & $3.4 \pm 0.6$ & $3.5 \pm 0.7$ & 0.48 \\
Cholesterol (mg per $100 \mathrm{ml})$ & $173 \pm 40$ & $164 \pm 39$ & 0.003 \\
Triglyceride (mg per $100 \mathrm{ml})$ & $158 \pm 143$ & $150 \pm 110$ & 0.325 \\
Creatinine (mg per $100 \mathrm{ml})$ & $10.8 \pm 4.0$ & $10.7 \pm 3.2$ & 0.835 \\
Uric acid (mg per $100 \mathrm{ml})$ & $7.4 \pm 1.5$ & $7.7 \pm 1.6$ & 0.087 \\
C-reactive protein $(\mathrm{mg} \mathrm{per} 100 \mathrm{ml})$ & $0.7 \pm 1.1$ & $1.4 \pm 2.1$ & 0.001 \\
Kt/V urea & $1.71 \pm 0.33$ & $1.66 \pm 0.31$ & 0.081 \\
Calcium (mg per $100 \mathrm{ml})$ & $9.6 \pm 4.0$ & $9.2 \pm 1.0$ & 0.195 \\
Phosphate (mg per $100 \mathrm{ml})$ & $4.9 \pm 1.8$ & $5.2 \pm 1.8$ & 0.156 \\
i-PTH (pg ml-1) & $231 \pm 311$ & $236 \pm 283$ & 0.456 \\
\hline
\end{tabular}

Abbreviations: i-PTH, intact parathyroid hormone; VAT, vascular access thrombosis.

previous studies. ${ }^{7,8,17,18}$ The VAT-free survival curve according to PP is illustrated in Figure 2. Patients with a $\mathrm{PP}>60 \mathrm{~mm} \mathrm{Hg}$ were more at risk for VAT than were patients with a $\mathrm{PP}<60 \mathrm{~mm} \mathrm{Hg}(P<0.001$, log-rank test).

The parameters that were significantly different between patients with and without VAT were further analyzed using univariate Cox 


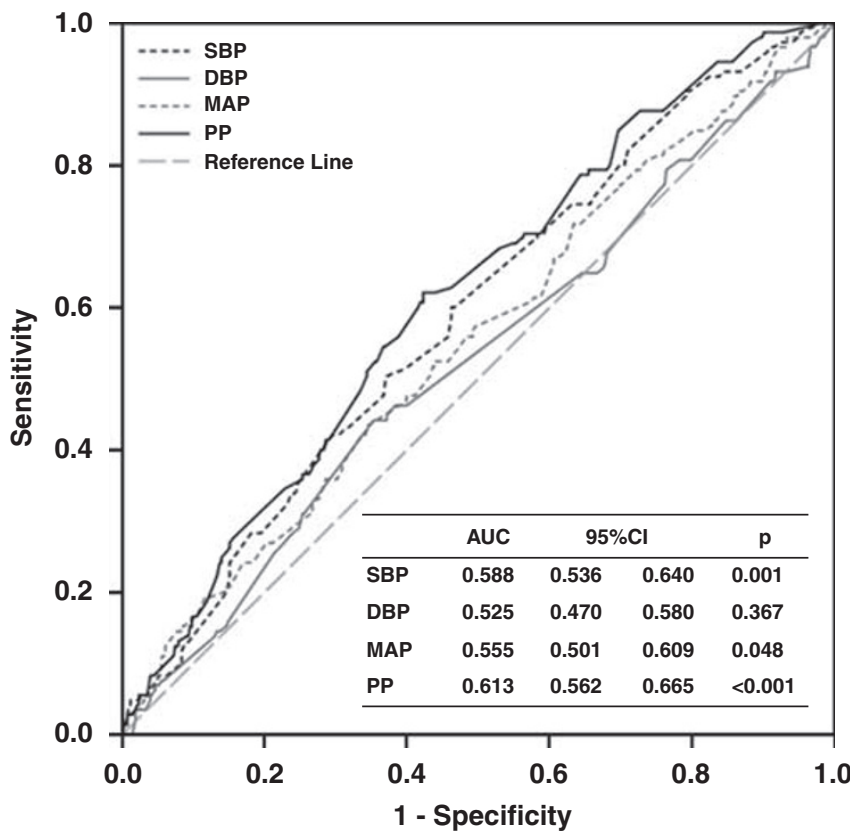

Figure 1 Receiver-operating characteristic curve for blood pressure measurements, including systolic blood pressure (SBP), diastolic blood pressure (DBP), mean arterial pressure (MAP) and pulse pressure (PP), in association with vascular access thrombosis.

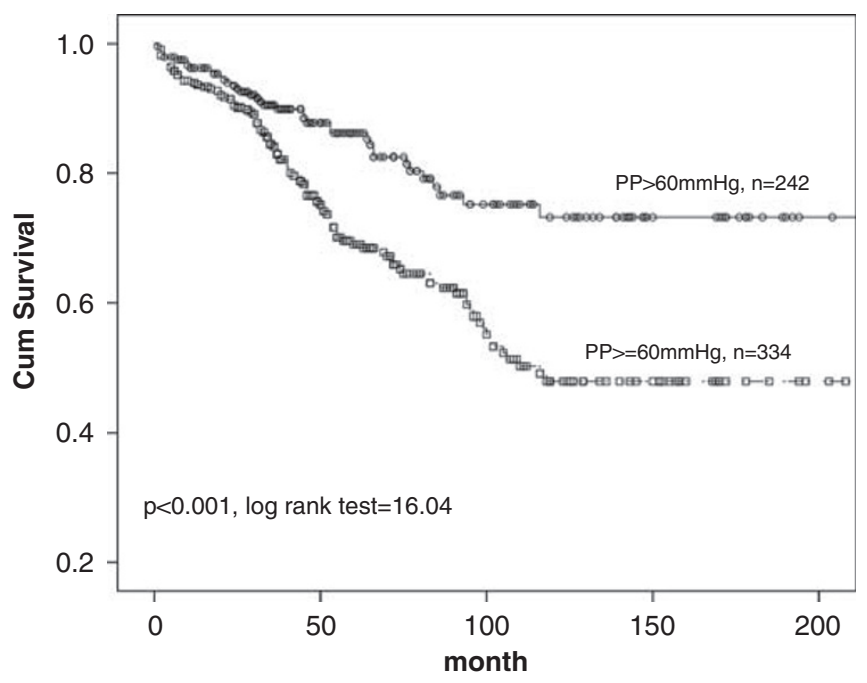

Figure 2 The Kaplan-Meier estimate for the vascular access thrombosis (VAT)-free survival curve in patients with pulse pressure $>$ and $<60 \mathrm{~mm} \mathrm{Hg}$.

regression. The variables used were gender and age, which were borderline significant, and hypertension and coronary arterial disease, which are well-known risk factors for VAT. ${ }^{1}$ The following hazard ratios (HRs) of possible risk factors are shown in Table 3: age $(P<0.001)$, SBP $(P=0.002)$, PP $(P<0.001)$, diabetes $(P<0.001)$, hypertension $(P=0.008)$, CRP $(P=0.001)$, warfarin $(P<0.001)$ and vascular access types $(P<0.001)$ were found to significantly predict the development of VAT. SBP was positively related to $\mathrm{PP}^{6,7,17}$ and was adjusted in multivariate Cox regression. The method of vascular access was an important predictor of VAT, as patients with a forearm nAVF
Table 3 HR of possible risk factors in univariate Cox regression

\begin{tabular}{lllr}
\hline & $H R$ & $(95 \% \mathrm{Cl})$ & P-value \\
\hline Age (years) & 1.02 & $(1.01-1.04)$ & $<0.001$ \\
Male & 1.28 & $(0.92-1.77)$ & 0.144 \\
SBP (every 10-mm Hg increase) & 1.17 & $(1.06-1.30)$ & 0.002 \\
PP (every 10-mm Hg increase) & 1.31 & $(1.15-1.50)$ & $<0.001$ \\
Diabetes & 2.09 & $(1.51-2.92)$ & $<0.001$ \\
HTN & 1.56 & $(1.51-2.92)$ & 0.008 \\
CAD & 1.36 & $(0.91-2.02)$ & 0.13 \\
Upper arm vs forearm nAVF & 2.94 & $(1.87-4.62)$ & $<0.001$ \\
AVG vs forearm nAVF & 3.42 & $(2.32-5.05)$ & $<0.001$ \\
Warfarin & 1.52 & $(1.02-2.76)$ & 0.02 \\
Cholesterol & 0.996 & $(0.992-1.001)$ & 0.088 \\
C-reactive protein & 1.18 & $(1.07-1.30)$ & 0.001 \\
(every 1-mg per 100 ml increase) & & & \\
\hline
\end{tabular}

Abbreviations: AVG, arteriovenous graft; CAD, coronary artery disease; HR, hazard ratio; HTN, hypertension; nAVF, native arteriovenous fistula.

Vascular access types are classified as forearm nAVF, upper-arm nAVF and AVG.

Table 4 HR of possible risk factors in multivariate Cox regression with adjustments for age, systolic blood pressure and vascular access types

\begin{tabular}{lccl} 
& $H R$ & $(95 \% \mathrm{Cl})$ & P-value \\
\hline PP (every 10-mm Hg increase) & 2.57 & $(1.50-4.40)$ & 0.001 \\
Diabetes & 1.80 & $(0.97-3.09)$ & 0.06 \\
HTN & 1.49 & $(0.90-2.46)$ & 0.12 \\
C-reactive protein (every 1-mg per $100 \mathrm{ml}$ increase) & 1.14 & $(1.01-1.27)$ & 0.017 \\
Warfarin & 1.06 & $(0.98-1.26)$ & 0.07
\end{tabular}

Abbreviations: AVG, arteriovenous graft; $\mathrm{Cl}$, confidence interval; HTN, hypertension; HR, hazard ratio; $n A V F$, native arteriovenous fistula; $\mathrm{PP}$, pulse pressure.

Vascular access types are classified as forearm nAVF, upper-arm nAVF and AVG.

were at a lowest risk for VAT, followed by those with an upper-arm nAVF and an AVG. The risk of VAT was not significantly different between patients with forearm AVG or upper-arm AVG $(P=0.79)$. For adjustments in multivariate Cox regression, the method of vascular access was stratified as forearm nAVF, upper-arm nAVF and AVG. The HRs of multivariate Cox regression with adjustments for age, SBP and the method of vascular access are shown in Table 4. Every 10-mm Hg increment of PP was associated with an HR of 2.57 ( $95 \%$ confidence interval: 1.5-4.4, $P=0.001$ ). In the multivariate Cox regression adjusted for age, SBP and the method of vascular access, the HR for patients with a $\mathrm{PP}>60 \mathrm{~mm} \mathrm{Hg}$ was 1.77 (95\% confidence interval: 1.08-2.92, $P=0.024$ ), suggesting that patients with a $\mathrm{PP}>60 \mathrm{~mm} \mathrm{Hg}$ had a 77\% increased risk of developing VAT compared with patients with a $\mathrm{PP}<60 \mathrm{~mm} \mathrm{Hg}$. Serum CRP independently predicted VAT, with an HR of 1.14 (95\% confidence interval: 1.01-1.27, $P=0.017$ ).

\section{DISCUSSION}

We found that PP was associated with the development of VAT in chronic HD patients and that the effect was independent of serum CRP levels. This was supported by two major findings. First, an increase in PP of $10 \mathrm{~mm} \mathrm{Hg}$ was associated with a 2.57-fold increase in VAT risk. Second, patients with a PP $>60 \mathrm{~mm} \mathrm{Hg} \mathrm{had} \mathrm{a} 77 \%$ increase in VAT risk compared with patients with a $\mathrm{PP}<60 \mathrm{~mm} \mathrm{Hg}$. The increase in PP has been found to be associated with vascular deformations, ${ }^{19}$ and reducing PP may normalize small-artery struc- 
ture. ${ }^{20}$ As vascular intimal hyperplasia and medial thickening are the major causes of VAT, the association between PP and VAT may be explained by the development of intimal hyperplasia and medial thickening in patients with increasing PP. We also found that serum CRP was positively associated with PP $(r=0.619, P=0.032$, Pearson's correlation). An elevated PP has been shown to be associated with an increase in indicators for inflammation. ${ }^{2,3,17,21,22}$ Elevated PP could induce an inflammatory state and increase the risk for atherosclerosis. ${ }^{15,21,23}$ On the basis of mounting evidence that suggests that VAT is an inflammatory condition, ${ }^{2,3,5}$ this may explain the increasing risk for VAT in patients with higher PP.

The anatomical location of vascular access is very important for the long-term patency of the vascular access point. In general, the survival of patients with a forearm nAVF was more favorable than that of patients with an upper-arm nAVF, followed by that of patients with an AVG. ${ }^{24-26}$ Approximately $90 \%$ of our HD patients had an nAVF, and patients with a primary upper-arm nAVF or AVG had poor vascular access preservation at the beginning of HD. It is not surprising that patients with upper-arm nAVFs or AVGs are more at risk for VAT than those with forearm nAVFs. The results from the unadjusted Cox regression model showed that upper-arm nAVF, forearm AVG and upper-arm AVG were associated with a 3.375-, 3.528- and 3.563-fold increase in the risk of VAT compared with those with a forearm nAVF. We stratified the method of vascular access in the multivariate Cox regression because the VAT risk was not significantly different among patients with forearm and upper-arm AVGs. In addition, warfarin treatment was associated with an increased VAT risk (Table 3); however, this was not found to be statistically significant in the adjusted Cox regression. Most of the patients received warfarin treatment in our study because of an underlying disease, such as deep vein thrombosis or atrial fibrillation. This finding may be explained by the association between underlying disease and VAT.

In a subgroup analysis of the REASON study, angiotensin-converting enzyme inhibitor was correlated with a diuretic decrease in PP, especially in patients with a baseline CRP $>3 \mathrm{mgl}^{-1} \cdot{ }^{27}$ Similarly, Christensen ${ }^{20}$ had suggested that reducing PP in hypertension may normalize the small-artery structure. Our findings (Table 4) partially support the role of PP-related inflammation indicated by increasing CRP levels in VAT patients. Owing to the lack of a cause-and-effect relationship between elevated PP and increasing CRP levels in our study, it is difficult to conclude that elevated PP results in inflammation and VAT. As PP is associated with inflammation and VAT, more studies are needed to explore whether a lower PP can decrease the risk of VAT.

In conclusion, an elevated PP was associated with the development of VAT among chronic HD patients with a functional vascular access point. The association between PP and VAT was independent of serum CRP levels. More interventional studies are needed to determine if treatment that decreases PP can decrease the risk of VAT among chronic HD patients.

\section{CONFLICT OF INTEREST}

The authors declare no conflict of interest.

1 Roy-Chaudhury P, Kelly BS, Zhang J, Narayana A, Desai P, Melham M, Duncan H, Heffelfinger SC. Hemodialysis vascular access dysfunction: from pathophysiology to novel therapies. Blood Purif 2003; 21: 99-110.
2 Chou CY, Kuo HL, Yung YF, Liu YL, Huang CC. C-reactive protein predicts vascular access thrombosis in hemodialysis patients. Blood Purif 2006; 24: 342-346.

3 Sirrs S, Duncan L, Djurdjev O, Nussbaumer G, Ganz G, Frohlich J, Levin A. Homocyst(e)ine and vascular access complications in haemodialysis patients: insights into a complex metabolic relationship. Nephrol Dial Transplant 1999; 14: 738-743.

4 Chen TC, Wang IK, Lee CH, Chang HW, Chiou TT, Lee CT, Fang JT, Wu MS, Hsu KT, Yang CC, Wang PH, Chuang FR. Hyperhomocysteinaemia and vascular access thrombosis among chronic hemodialysis patients in Taiwan: a retrospective study. Int J Clin Pract 2006; 60: 1596-1599.

5 Chang CJ, Ko YS, Ko PJ, Hsu LA, Chen CF, Yang CW, Hsu TS, Pang JH. Thrombosed arteriovenous fistula for hemodialysis access is characterized by a marked inflammatory activity. Kidney Int 2005; 68: 1312-1319.

6 Klassen PS, Lowrie EG, Reddan DN, DeLong ER, Coladonato JA, Szczech LA, Lazarus JM, Owen Jr WF. Association between pulse pressure and mortality in patients undergoing maintenance hemodialysis. JAMA 2002; 287: 1548-1555.

7 Tozawa M, Iseki K, Iseki C, Takishita S. Pulse pressure and risk of total mortality and cardiovascular events in patients on chronic hemodialysis. Kidney Int 2002; 61: 717-726.

8 Safar ME, Blacher J, Pannier B, Guerin AP, Marchais SJ, Guyonvarc'h PM, London GM. Central pulse pressure and mortality in end-stage renal disease. Hypertension 2002; 39: 735-738.

9 Amar J, Vernier I, Rossignol E, Bongard V, Arnaud C, Conte JJ, Salvador M, Chamontin B. Nocturnal blood pressure and 24-h pulse pressure are potent indicators of mortality in hemodialysis patients. Kidney Int 2000; 57: 2485-2491.

10 Abramson JL, Weintraub WS, Vaccarino V. Association between pulse pressure and C-reactive protein among apparently healthy US adults. Hypertension 2002; 39: 197-202.

11 Chae CU, Lee RT, Rifai N, Ridker PM. Blood pressure and inflammation in apparently healthy men. Hypertension 2001; 38: 399-403.

12 Ishimitsu T, Nakano N, Sudo Y, Akashiba A, Takahashi T, Ohta S, Minami J, Matsuoka $\mathrm{H}$. Predictive significance of blood pressure values for the incidence of cardiovascular events in chronic hemodialysis patients. Hypertens Res 2008; 31: 1703-1709.

13 Zoccali C, Benedetto FA, Mallamaci F, Tripepi G, Fermo I, Foca A, Paroni R, Malatino LS. Inflammation is associated with carotid atherosclerosis in dialysis patients. Creed Investigators. Cardiovascular Risk Extended Evaluation in Dialysis Patients. J Hypertens 2000; 18: 1207-1213.

14 Agarwal R, Nissenson AR, Batlle D, Coyne DW, Trout JR, Warnock DG. Prevalence, treatment, and control of hypertension in chronic hemodialysis patients in the United States. Am J Med 2003; 115: 291-297.

15 Halcox JP, Schenke WH, Zalos G, Mincemoyer R, Prasad A, Waclawiw MA, Nour KR, Quyyumi AA. Prognostic value of coronary vascular endothelial dysfunction. Circulation 2002; 106: 653-658.

16 Schwaiger JP, Lamina C, Neyer U, Konig P, Kathrein H, Sturm W, Lhotta K, Grochenig $\mathrm{E}$, Dieplinger $\mathrm{H}$, Kronenberg $\mathrm{F}$. Carotid plaques and their predictive value for cardiovascular disease and all-cause mortality in hemodialysis patients considering renal transplantation: a decade follow-up. Am J Kidney Dis 2006; 47: 888-897.

17 Foucan L, Deloumeaux J, Hue K, Foucan T, Blanchet-Deverly A, Merault H, Gabriel JM, Hiesse $C$. High pulse pressure associated with cardiovascular events in patients with type 2 diabetes undergoing hemodialysis. Am J Hypertens 2005; 18: 1457-1462.

18 Avanzini F, Alli C, Boccanelli A, Chieffo C, Franzosi MG, Geraci E, Maggioni AP, Marfisi RM, Nicolosi GL, Schweiger C, Tavazzi L, Tognoni G, Valagussa F, Marchioli R. High pulse pressure and low mean arterial pressure: two predictors of death after a myocardial infarction. J Hypertens 2006; 24: 2377-2385.

19 Dobrin PB. Mechanical factors associated with the development of intimal and medial thickening in vein grafts subjected to arterial pressure: a model of arteries exposed to hypertension. Hypertension 1995; 26: 38-43.

20 Christensen KL. Reducing pulse pressure in hypertension may normalize small artery structure. Hypertension 1991; 18: 722-727.

21 Tsai JC, Kuo HT, Chiu YW, Hwang SJ, Chuang HY, Chang JM, Chen HC, Lai YH. Correlation of plasma homocysteine level with arterial stiffness and pulse pressure in hemodialysis patients. Atherosclerosis 2005; 182: 121-127.

22 Abramson JL, Vaccarino V. Pulse pressure and inflammatory process in atherosclerosis. Adv Cardiol 2007; 44: 223-233.

23 Karakitsos D, Patrianakos AP, De Groot E, Boletis J, Karabinis A, Kyriazis J, Samonis G, Parthenakis FI, Vardas PE, Daphnis E. Androgen deficiency and endothelial dysfunction in men with end-stage kidney disease receiving maintenance hemodialysis. Am J Nephrol 2006; 26: 536-543.

24 Berardinelli L, Vegeto A. Lessons from 494 permanent accesses in 348 haemodialysis patients older than 65 years of age: 29 years of experience. Nephrol Dial Transplant 1998; 13(Suppl 7): 73-77.

25 Dixon BS, Novak L, Fangman J. Hemodialysis vascular access survival: upper-arm native arteriovenous fistula. Am J Kidney Dis 2002; 39: 92-101.

26 Erkut B, Unlu Y, Ceviz M, Becit N, Ates A, Colak A, Kocak H. Primary arteriovenous fistulas in the forearm for hemodialysis: effect of miscellaneous factors in fistula patency. Ren Fail 2006; 28: 275-281

27 Amar J, Ruidavets JB, Peyrieux JC, Mallion JM, Ferrieres J, Safar ME, Chamontin B. C-reactive protein elevation predicts pulse pressure reduction in hypertensive subjects. Hypertension 2005; 46: 151-155. 